\title{
DESENVOLVIMENTO DE UM MODELO DE APLICATIVO PARA CELULAR DO TRANSPORTE URBANO COLETIVO DA CIDADE DE SÃO JOSÉ DOS CAMPOS - SÃO PAULO.
}

\author{
J.A.A. Neto ${ }^{1,}{ }^{*} ;$ N.G. Amorim ${ }^{1}$ \\ 1 Faculdade de Tecnologia de São José dos Campos - Professor Jessen Vidal \\ Av. Cesare Mansueto Giulio Lattes, 1350 - Eugênio de Melo, São José dos Campos/SP, \\ CEP.: 12247-014, Brasil. \\ Telefone: (12) 3905-2423 \\ *jose.alvarenga01@fatec.sp.gov.br
}

\begin{abstract}
RESUMO: O significativo aumento populacional nas cidades torna a mobilidade urbana um tema amplamente discutido, já que é um atributo das cidades e está relacionado ao ir e vir de pessoas e bens dentro dos centros urbanos. Este artigo tem o objetivo de melhorar a mobilidade urbana, buscando tornar o transporte público coletivo atrativo aos não usuários. Foram feitas pesquisas bibliográficas, além de análise conjunta e análise de benchmarking de aplicativos já desenvolvidos para definir um modelo apropriado às necessidades dos usuários e não usuários do transporte. Chegando a um modelo onde tanto a visualização das informações quanto aos horários e localização do ônibus serão claros e eficientes.
\end{abstract}

PALAVRAS-CHAVE: Transporte Público; Mobilidade Urbana; Ônibus; Aplicativo.

ABSTRACT: The significant population increase in cities makes urban mobility a widely discussed theme, which is already an attribute of cities and is related to the coming and going of people and goods within urban centers. This article aims to improve urban mobility, seeking to make public transport attractive to non-users. Bibliographic searches were made, In addition to joint analysis and benchmarking analysis of applications already developed to define a model appropriate to the needs of non-transport user's needs. Coming to a model where both the visualization of information and the time and location of the bus will be clear and efficient.

KEYWORDS: Public Transport; Urban Mobility; Bus; App.

\section{INTRODUÇÃO}

O direito de locomoção, também conhecido como direito de ir e vir é garantido, por meio da Constituição Federal de 1988 onde todo cidadão tem liberdade de locomoção, por todo território nacional, sem restrições arbitrárias por parte dos Estados e Municípios a esta liberdade (BISSARO, 2017).

Para isto o desenvolvimento do planejamento urbano deve possuir um maior foco nas necessidades das pessoas que usam as cidades (GEHL apud GARCIA 2018)

Neste âmbito é que o planejamento, as estratégias e a logística na mobilidade urbana são mensurados com base na metodologia adotada pela política governamental que por sua vez sofrem diretamente com as mudanças no cenário (PARENTE et al, 2016). 
Contudo para estudar a mobilidade urbana deve-se reconhecer que o uso do espaço urbano faz parte de uma comunidade, que se ordenou em relação aos diversos tipos de deslocamentos de acordo com sua necessidade (BISSARO, 2017).

Neste sentido, a mobilidade urbana possui o desafio de, conforme descrito por Costa (2015, p.120): "Oferecer um transporte de qualidade e capaz de atender à demanda de deslocamentos atual e futura". Afinal, como relatado pelo instituto PÓLIS (2005), a mobilidade é mais do que o denominado transporte urbano que conhecemos, mas sim o resultado da interação entre os deslocamentos de pessoas e bens com a cidade. Exemplifica ainda como, a disponibilidade de meios e infraestrutura adequados para os deslocamentos de pessoas e bens numa área da cidade, pode ajudar a cidade a se desenvolver.

Para tanto, Costa (2015, p.120): relata que os países em desenvolvimento, que vivem, em geral, um processo de urbanização desordenado, enfrenta um problema complexo, onde, o uso do carro vem crescendo de maneira insustentável, tanto pela falta de qualidade no transporte público, quanto pela questão cultural do carro ser uma representação de status social. Complementa ainda, Vianna (2013, p.09): "A rápida urbanização do país não foi acompanhada no mesmo ritmo por investimentos de infraestrutura necessários, levando a constantes aumentos nos congestionamentos de trânsito das grandes cidades e deterioração das condições dos serviços públicos de transportes".

Para a locomoção de pessoas leva-se em conta o bem-estar, conforto e agilidade, economizando tempo no transporte, as pessoas no cenário atual perdem muito tempo estando no engarrafamento, mas isso não é a única coisa que se perde, mais também saúde tanto fica como emocional, dinheiro e em alguns casos até a vida (RUBIM, LEITÃO, 2013).

Se tratando do transporte público observa-se que muitos dos problemas enfrentados pela população são ônibus lotados, estes que, muitas vezes, não correspondem ao itinerário programado, tarifas elevadas, esses problemas diários não afetam apenas o conforto e o bem-estar, mais também o dia a dia da população (SILVEIRA, COCCO, 2013).

Neste contexto, apesar da existência de aplicativos atuais que apresentam o itinerário que o transporte coletivo deveria estar passando, os mesmos não são eficientes, já que diverge dos horários, o que torna relevante um estudo baseado no desenvolvimento de um novo modelo de software direcionado a este tema.

A natureza metodológica deste estudo é caracterizada como aplicada, pois se trata da estruturação de um modelo de aplicativo que objetiva a aplicação prática a partir de seu envio as partes competentes da prefeitura da cidade, além de envolver os interesses da cidade e possibilitar a melhora da comunicação entre as partes mais eficiente, afinal, Gerhardt e Silveira (2009, p.35): "Objetiva gerar conhecimentos para aplicação prática, dirigidos à solução de problemas específicos. Envolve verdades e interesses locais".

Por conseguinte, em referência aos objetivos de estudo, está caracteriza-se como exploratória, pois busca, a partir de um levantamento bibliográfico e benchmarking o melhor entendimento dos problemas relacionados com o tema em estudo, buscando assim construir hipóteses para solucionálos, neste contexto especificado por Gerhardt e Silveira (2009, p.32): "Este tipo de pesquisa tem como objetivo proporcionar maior familiaridade com o problema, com vistas a torná-lo mais explícito ou a construir hipóteses".

Neste contexto, é necessário entender, como parte relevante, o trato da abordagem, sendo está, aplicada a este estudo de forma combinada, onde a partir do benchmarking, serão subjetivados as divergências relacionadas entre a região em estudo e as regiões sob análise, incluindo, de maneira intrínseca, a interação do cidadão com o aplicativo a ser modelado. Como explica Gerhardt e Silveira (2009, p.32): "As características da pesquisa qualitativa são: objetivação do fenômeno; hierarquização das ações de descrever, compreender, explicar, precisão das relações entre o global e o local em determinado fenômeno". E ainda a "observância das diferenças entre o mundo social e o 
mundo natural" além de "respeito ao caráter interativo entre os objetivos buscados pelos investigadores, suas orientações teóricas e seus dados empíricos" por fim "busca de resultados os mais fidedignos possíveis; oposição ao pressuposto que defende um modelo único de pesquisa para todas as ciências".

Por fim, o método utilizado refere-se a um estudo de caso, pois trata-se do desenvolvimento de um modelo de aplicativo, objetivando a sua posterior aplicação por meio da prefeitura do município, afinal como descreve GIL apud a Gerhardt e Silveira (2009, p.39): "Um estudo de caso pode ser caracterizado como um estudo de uma entidade bem definida como um programa, uma instituição, um sistema educativo, uma pessoa, ou uma unidade social" informa ainda que "Visa conhecer em profundidade o como e o porquê de uma determinada situação que se supõe ser única em muitos aspectos, procurando descobrir o que há nela de mais essencial e característico" afirma ainda que "O pesquisador não pretende intervir sobre o objeto a ser estudado, mas revelá-lo tal como ele o percebe”.

\section{REVISÃO DA LITERATURA}

\subsection{Mobilidade Urbana}

Mobilidade urbana é conceituada como sendo um atributo das cidades que se refere ao deslocamento de pessoas e bens no espaço urbano, onde o deslocamento é realizado através de veículos automotores, vias, calçadas e toda a infraestrutura que é utilizada para o ir e vir da população (BRITO, PEREIRA, 2017).

Mas no atual cenário a melhor definição é, mobilidade indica a possibilidade e o grau da intensidade de locomoção, ou melhor, a facilidade com a qual cargas e pessoas se locomovem pelas vias públicas, no caso da população para a realização de atividades cotidianas e suas necessidades (MARRARA, 2015).

O objetivo da mobilidade urbana é levar as pessoas para o lugar certo, no tempo certo, isso só é possível com a adequada locomoção que gera oportunidades a sociedade, sem priorizar um grupo, mais também é objetivo da mesma garantir o menor custo de viagem, segurança e comodidade as pessoas (JUNIOR apud BRITO, PEREIRA, 2017).

E um dos problemas enfrentados pela sociedade é transitar pela cidade de modo ágil e seguro no seu dia a dia, isso se dá pelo crescimento acelerado das cidades e o mau planejamento das políticas de mobilidade urbana (ANDRADE, CUNHA, 2017).

E para melhor satisfazer as necessidades da população em relação a mobilidade urbana é ter um bom planejamento urbano, onde será abordado os principais problemas e sempre visando a qualidade de vida da população. (REIS, 2014).

\subsection{Transporte Público}

Um dos objetivos do transporte público é proporcionar qualidade de vida para a população com o serviço oferecido, facilitando o acesso aos lugares das cidades, o local de serviço, de lazer, acesso a saúde, e de cultura. Mas nem sempre isso é uma tarefa fácil já que no Brasil a infraestrutura não favorece o transporte e a mobilidade, o que torna com que o objetivo seja difícil de ser alcançado (SILVEIRA, COCCO, 2013).

Segundo o autor uma das deficiências desse serviço são os investimentos nos equipamentos de transporte e movimentação que são muito baixas em relação a quantidade de usuários que necessitam utilizar desses serviços. E se tratando do transporte público não só a tarifa que não se adequada, pois a qualidade do serviço prestado não é a esperada, o sistema não é eficaz, o que não atraí o público a utilizar do mesmo.

O transporte coletivo tem grande importância para o processo de gerenciamento da cidade, e quando se tem um modelo adequado os benefícios que se tem é a redução dos congestionamentos, 


\section{ISSN $2447-5378$}

riscos de acidentes e assim a qualidade de vida dos cidadãos é alta (LOMBARDO, CARDOSO, SOBREIRA, 2012).

\subsection{Aplicativos para Celular}

Com a evolução proveniente dos grandes avanços da tecnologia é percebido a criação de uma nova era digital, que com o crescimento explosivo nas áreas da informática, telecomunicações, informação, transporte e outras tecnologias geraram um grande impacto no modo como as empresas entregam valor aos clientes (KOTLER apud CARDIAS, VALENTE et al, 2017).

Neste sentido, Castro, Tedesco apud Hino, Cunha (2014) apontam as tecnologias móveis como o fator que propiciou a mudança do relacionamento homem-tecnologia, sendo que permitem a flexibilidade, pois seus usuários podem leva-las onde quiserem, explorando esta oportunidade é possível atender demandas antes impossíveis.

Sendo, facilitados pelos aplicativos mobile que, por sua vez, são segundo Miller, Matviyenko apud Lima (2017) pequenos programas, que por sua vez compõem como parte de um software que são programados para usar de maneira especifica e particular o poder uma de um sistema de computação.

Para tanto, esta evolução tem gerado aos poucos uma mudança da sociedade na maneira de pensar, agir e de principalmente se comunicar (CARDIAS, VALENTE et al, 2017).

Neste contexto, e atualmente, é percebido que se tornou comum para as famílias a reunião por meio de grupos no Whatsapp, amigos conectados pelo Facebook, que compras sejam feitas por aplicativos de lojas, informações cheguem a todos os momentos por meio de sites, redes sociais entre outros (LIMA, 2017)

Pois desde que o ser humano passou a se vale da fala até o momento que utiliza equipamentos moveis para acessar a rede mundial de computadores, houve diversas mudanças na forma da comunicação para as relações sociais (CARDOSO, SANTOS e VARGAS apud CADIAS, VALENTE et al, 2017).

Neste sentido, para comtemplar estas comunicações sociais é necessário desenvolver também esta interação com os governos, sendo os aplicativos móveis uma potencial ferramenta de cidadania, inseridas a uma nova modalidade de governo eletrônico (LIMA, 2017).

Afinal, com o desenvolvimento de uma cultura digital, é esperado pela sociedade que os governos possuam o mesmo nível de agilidade e conectividade que encontram no relacionamento com empresas privadas (LIMA, 2017).

\subsubsection{Aplicativos para Transporte Público.}

A evolução da tecnologia de acordo com Lima (2017) permitiu a sociedade identificar em redes e dispositivos novas possibilidades para suas necessidades de interação e execução de tarefas, possibilidades estas também buscadas pela administração publica por meio das tecnologias de informação e comunicação (TIC), pois se faz necessário encontrar soluções para o relacionamento entre governos e cidadãos, visando à melhoria da atuação do estado.

Neste aspecto os aplicativos de mobilidade objetivam a facilitação da vida dos usuários, pois executam atividades como traçar rotas, apresentar informações de tráfego, pedágio e pontos de referência bem como horários de chegada de transporte público (ALMEIDA, MESQUITA et al apud HINO, CUNHA, 2018).

Estas tecnologias trazem a possibilidade para mudar forma como o setor público funciona, em relação ao desempenho institucional, capazes de aumentar a transparência, a participação, a colaboração e a capacidade de resposta, assemelhado ao que ocorreu no setor privado visando produzir uma maior eficiência e eficácia (WEST apud LIMA, 2017). 
Neste sentido Da Silva, Urssi apud Hino, Cunha (2018) cita que estes aplicativos facilitam a vida das pessoas em ambientes urbanos, sendo que os benefícios e motivos que incentivam o uso desses aplicativos está na agilidade do serviço, otimização do deslocamento que contribuem para a redução do tempo de mobilidade, além do melhor controle do processo, pois os usuários dos aplicativos podem possuir mais informação de como, onde e quando chegar ao local desejado.

Sendo assim, além de agregar valor aos usuários os dispositivos móveis possuem alta penetração o que torna relevante a adoção destes aplicativos pelo setor publico, possibilitando assim o atendimento das demandas dos cidadãos por eficiência e ganhos de produtividade por meio da mobilidade (KUSHCHU apud LIMA, 2017).

Para isto, nos aplicativos relacionados a mobilidade, os usuários não buscam apenas as rotas, mas também informações como congestionamentos, velocidades médias, radares, segurança nas vias entre outros (FRANÇOZO, MELLO apud HINO, CUNHA 2018).

Por fim é valido notar que esta evolução oferece uma infraestrutura social reconfortante para a sociedade, pois estes aplicativos, respiram, processam e expiram dados que existem ao nosso redor (KUSHCHU apud LIMA 2017).

Por fim é valido notar que esta evolução oferece uma infraestrutura social reconfortante para a sociedade, pois estes aplicativos, respiram, processam e expiram dados que existem ao nosso redor (KUSHCHU apud LIMA 2017).

\section{DESENVOLVIMENTO}

Inicialmente, delimitou-se o problema do transporte publico coletivo da cidade de São José dos Campos, São Paulo para a área em estudo, que se trata diretamente do uso do aplicativo para celular, sendo este um fator essencial para os tempos atuais por se tratar da integração das pessoas com o transporte e principalmente por se relacionar com a comodidade e facilidade para o usuário em sua utilização.

Além do crescente uso dos celulares pela população nos dias de hoje ser um importante critério a ser observado, foi percebido também que o objetivo do município de reduzir o trafego nas áreas urbanas da cidade se caracteriza como uma relevante característica parar entender como está à integração do aplicativo com o transporte, dado que as pessoas buscam comodidade e principalmente rapidez quando optam pelo meio de locomoção que irão fazer uso.

Afinal, optar ou não pelo uso do transporte urbano coletivo decorre de uma serie de fatores, e ter clareza nas informações gera um diferencial neste momento, portanto para contribuir com o objetivo do município em questão é necessário entender principalmente o que pessoas que não fazem o uso deste meio de locomoção esperam de um aplicativo deste gênero, para assim gerar atratividade em seu uso.

Neste contexto, foi realizada uma pesquisa de campo que objetivou entender o que os munícipes esperam de um aplicativo para o transporte publico urbano, e principalmente, através de benchmarking, qual melhor modelo seria utilizado para atender as suas necessidades.

Esta pesquisa foi baseada em diversas características de entrada (Ações necessárias de execução por parte do usuário, ou seja, são as informações inseridas no aplicativo) e saída (São as informações prestadas pelo aplicativo com base nos dados imputados pelo usuário) de aplicativos de transporte, sendo Waze, Uber e 99, basicamente por se tratarem dos mais utilizados para a área relatada e também Moovit e Cittamobi por serem os melhores e mais próximos da região, por fim o aplicativo Ônibus na hora SJC atualmente utilizado no sistema atual de transporte coletivo da cidade.

As questões abordadas nesta pesquisa foram:

Como deveria ser o aplicativo de ônibus? 
O que deveria ter no aplicativo do ônibus de São José dos Campos - SP?

1) Qual a sua faixa etária?*

- Menor de 18 anos

- Entre 18 e 25 anos

- Entre 25 e 60 anos

- Mais de 60 anos

2) Com qual frequência utiliza o serviço de transporte público da cidade? *

- De vez em quando

- Frequente

- Não utiliza

3) Selecione 03 opções que você julga mais importante: o que tem que aparecer ao abrir o aplicativo? *

- Onde estou

- Para onde vou

- Pontos de ônibus próximos

- A Imagem dos veículos na região

- Seus locais favoritos

4) Selecione 04 opções que você julga mais importante: o que o aplicativo tem que informar? *

- Localização do ônibus

- Quando o ônibus vai chegar

- Tempo aproximado de viagem

- Trajeto em tempo real

- Quais ônibus deve pegar para chegar em seu destino

- Quando pegar ônibus e quando ir a pé

- Informação do Ônibus atrasado

A partir dos resultados desta pesquisa, cujo número de respondentes foi de 85 pessoas, foi realizada uma comparação a partir de benchmarking com os principais aplicativos para celular em relação aos aplicativos de transporte e o de transporte publico coletivo utilizado atualmente na região.

Neste caso, primeiramente foi confrontado as informações de entrada, tendo em vista que as áreas demarcadas com um " $\mathrm{X}$ " se equivalem aos critérios presentes no aplicativo em questão e também a informação "\% Total" é a porcentagem que aquele aplicativo possui em relação ao atendimento das sugestões dos participantes da pesquisa enquanto "\% Não Utiliza" se refere a quanto o aplicativo em questão atende as sugestões dos participantes da pesquisa que não utilizam o transporte publico coletivo, conforme mostra a Tabela 1. 
Tabela 1 - Análise de Benchmarking (Entradas).

\begin{tabular}{|c|c|c|c|c|c|c|}
\hline Entrada & Waze & Uber & 99 & Moovit & Ônibus na Hora SJC & Cittamobi \\
\hline Onde estou & $\mathrm{X}$ & $\mathrm{X}$ & $\mathrm{X}$ & $\mathrm{X}$ & $\mathrm{X}$ & $\mathrm{X}$ \\
\hline Para onde vou & $\mathrm{X}$ & $\mathrm{X}$ & $\mathrm{X}$ & $\mathrm{X}$ & $\mathrm{X}$ & $\mathrm{X}$ \\
\hline Pontos de ônibus próximos & & & & $\mathrm{X}$ & & $\mathrm{X}$ \\
\hline A Imagem dos veículos na região & $\mathrm{X}$ & $\mathrm{X}$ & $\mathrm{X}$ & & & \\
\hline Seus locais favoritos & $\mathrm{X}$ & $\mathrm{X}$ & $\mathrm{X}$ & $\mathrm{X}$ & $\mathrm{X}$ & $\mathrm{X}$ \\
\hline \% Total & $71 \%$ & $71 \%$ & $71 \%$ & $93 \%$ & $64 \%$ & $93 \%$ \\
\hline \%ão Utiliza & $79 \%$ & $79 \%$ & $79 \%$ & $\mathbf{8 2} \%$ & $62 \%$ & $82 \%$ \\
\hline
\end{tabular}

Analisando estes dados é possível perceber que quanto as informações de entrada sugeridas o aplicativo atual da cidade atende apenas a $62 \%$ das sugestões de quem não utiliza o transporte, enquanto nos demais existe uma porcentagem de $82 \%$ neste mesmo quesito nos aplicativos concorrentes.

Por conseguinte verificaram-se os critérios de saída onde com as informações assemelhadas dos pontos de entrada foram analisados conforme as sugestões totais da pesquisa e logo após dos não usuários, como demostra a Tabela 2.

Tabela 2 - Análise de Benchmarking (Saídas).

\begin{tabular}{|c|c|c|c|c|c|c|}
\hline Saída & Waze & Uber & 99 & Moovit & Ônibus na Hora SJC & Cittamobi \\
\hline Localização do ônibus & $\mathrm{X}$ & $\mathrm{X}$ & $\mathrm{X}$ & & & $\mathrm{X}$ \\
\hline Quando o ônibus vai chegar & $\mathrm{X}$ & $\mathrm{X}$ & $\mathrm{X}$ & $\mathrm{X}$ & $\mathrm{X}$ & $\mathrm{X}$ \\
\hline Tempo aproximado de viagem & $\mathrm{X}$ & $\mathrm{X}$ & $\mathrm{X}$ & $\mathrm{X}$ & $\mathrm{X}$ & $\mathrm{X}$ \\
\hline Trajeto em tempo real & $\mathrm{X}$ & $\mathrm{X}$ & $\mathrm{X}$ & $\mathrm{X}$ & & \\
\hline Quais ônibus deve pegar para chegar em seu destino & $\mathrm{X}$ & & & & $\mathrm{X}$ & \\
\hline Quando pegar ônibus e quando ir a pé & & & & $\mathrm{X}$ & & $\mathrm{X}$ \\
\hline Informação do Ônibus atrasado & $\mathrm{X}$ & $\mathrm{X}$ & $\mathrm{X}$ & & & $\mathrm{X}$ \\
\hline$\%$ Total & $97 \%$ & $80 \%$ & $80 \%$ & $51 \%$ & $52 \%$ & $69 \%$ \\
\hline \% Não Utiliza & $96 \%$ & $71 \%$ & $71 \%$ & $65 \%$ & $59 \%$ & $51 \%$ \\
\hline
\end{tabular}

Neste caso é percebido que o "aplicativo Ónibus na Hora SJC" atende, no total geral, apenas $59 \%$ dos critérios dos não usuários, enquanto o aplicativo Waze já alcança $96 \%$ dos pontos levantados nesta pesquisa quanto aos que não fazem uso do transporte publico coletivo.

Por fim, de uma forma geral compilou-se ambas as informações presentes na pesquisa buscando entender de uma maneira completa um atendimento total de cada aplicativo as sugestões dos respondentes, sendo, neste caso, "\% Total Entrada e saída" como o percentual de atendimento aos critérios do total dos respondentes, enquanto "\% Total Entrada e saída (Não utiliza)" refere-se à mesma informação, porém baseando-se nos não usuários, informado pela Tabela 3.

Tabela 3 - Análise de Benchmarking.

\begin{tabular}{|r|c|c|c|c|c|c|}
\hline \% Total Entrada e saida & $86 \%$ & $76 \%$ & $76 \%$ & $69 \%$ & $57 \%$ & $79 \%$ \\
\hline \% Total Entrada e saida (Não utiliza) & $87 \%$ & $75 \%$ & $75 \%$ & $74 \%$ & $60 \%$ & $66 \%$ \\
\hline
\end{tabular}

Portanto, em uma análise final notou-se que de maneira geral o aplicativo possui um baixo atendimento quando comparado aos demais aplicativo, tendo como exemplo o aplicativo Waze que possui um percentual de $87 \%$ enquanto o utilizado na região apenas $60 \%$ dos não usuários de transporte, tendo então baixa atratividade para estes, o que desvia do objetivo do município, sendo então base para o desenvolvimento de um modelo que seja capaz de atende-los de maneira eficiente.

Sendo assim, a partir destas informações foram relacionados dentre os aplicativos utilizados na pesquisa suas características cujo atendimento as sugestões fossem compatíveis e para um maior 


\section{ISSN $2447-5378$}

entendimento de como o aplicativo irá funcionar foi criado um modelo de aplicativo com uma simulação.

Uma pessoa que está localizada no bairro A de uma cidade e precisa chegar no bairro B, para chegar até o seu destino irá utilizar o transporte público coletivo, porém esta não conhece a cidade e principalmente quais ônibus deverá utilizar.

Neste caso irá fazer uso do aplicativo de ônibus da cidade, onde ao abrir o aplicativo será solicitado o uso de internet e de GPS (Sistema de Posicionamento Global), e com isso irá surgir a tela com a sua localização atual.

Com isso será solicitado ao usuário seu destino desejado, que neste caso está localizado no bairro B. E assim será informado pelo aplicativo qual o ponto de ônibus a ser utilizado a sua localização, quais ônibus deverá pegar e qual o tempo de viagem, respectivamente representado na Tela 1 e Tela 2.

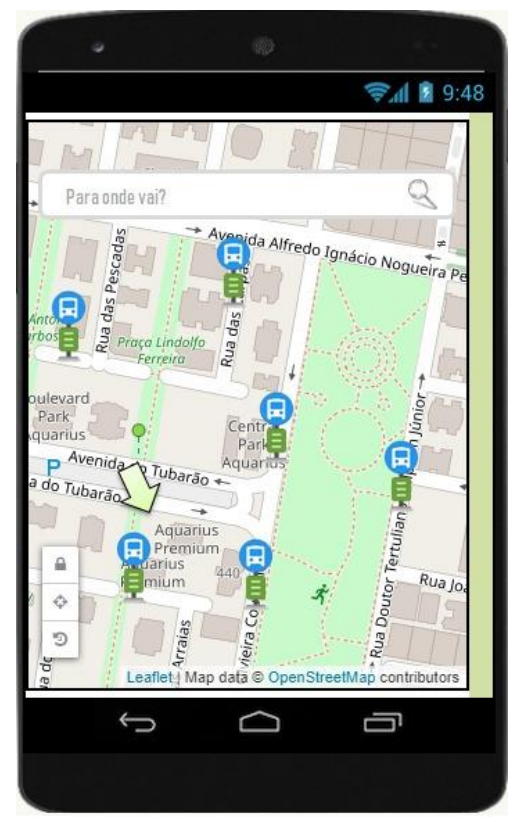

Tela 1. Aplicativo

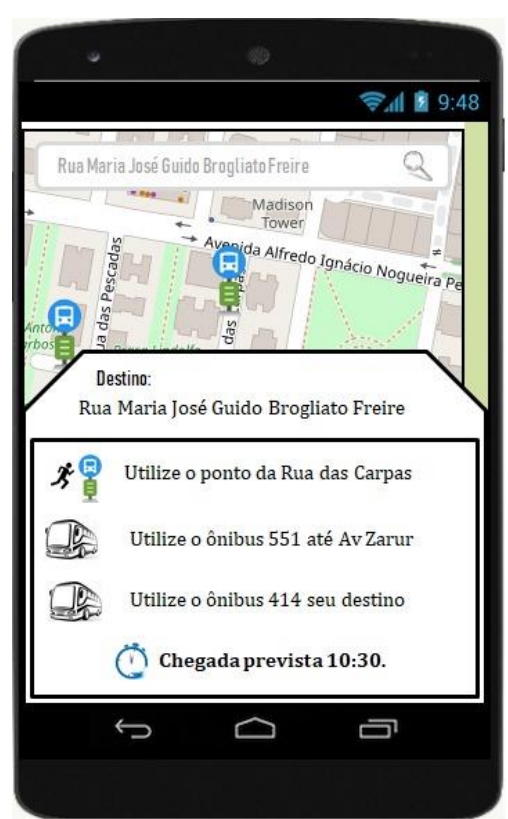


Tela 2. Aplicativo

Os próximos passos, Tela 3 e Tela 4 respectivamente, será sinalizado ao usuário que se dirija até o ponto de ônibus destacado tendo informado pelo aplicativo o tempo médio até o ponto. Ao chegar no ponto será informado o horário que o ônibus vai passar e sua localização em tempo real, ao adentrar ao ônibus será mostrado o trajeto em tempo real e também informará em qual ponto o usuário deverá descer e, logo após, para qual ponto deve se dirigir para realizar a baldeação e também o horário que esse segundo ônibus irá passar, demostrando de forma clara todas as informações em tempo real.

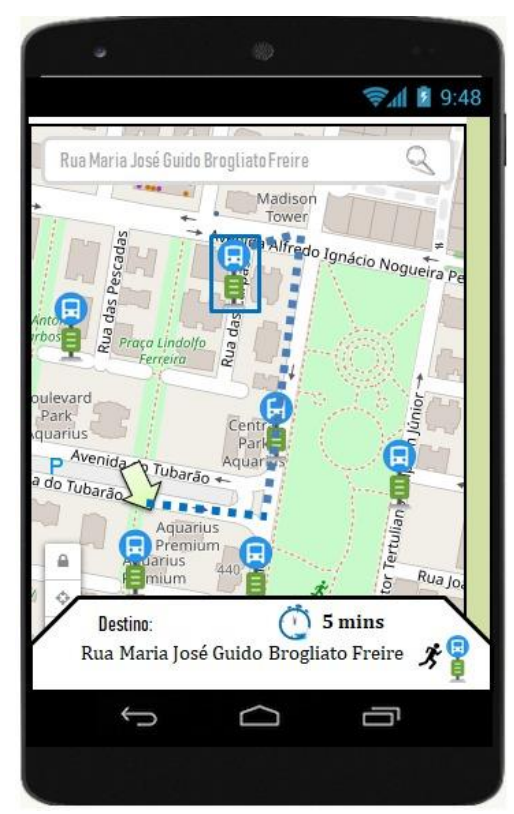

Tela 3. Aplicativo

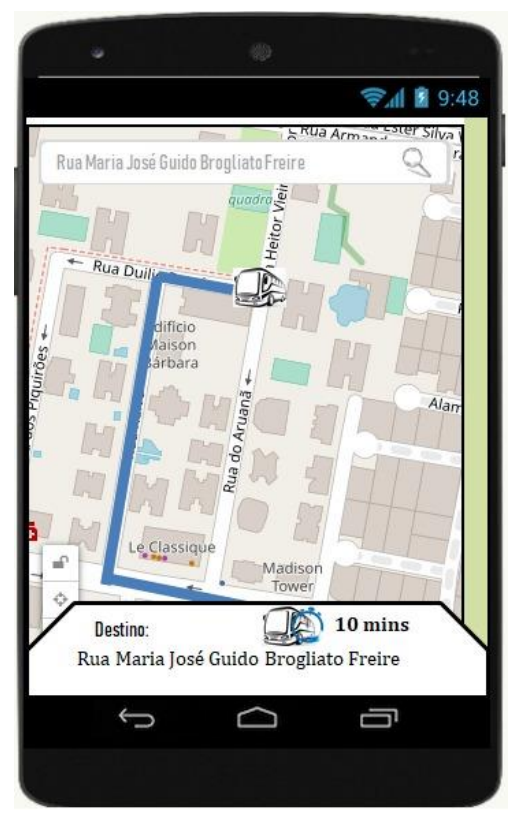

Tela 4. Aplicativo 
Já no último passo Tela 5 será informado em qual ponto o usuário irá descer e se deverá caminhar até o seu destino, finalizando assim o uso do aplicativo.

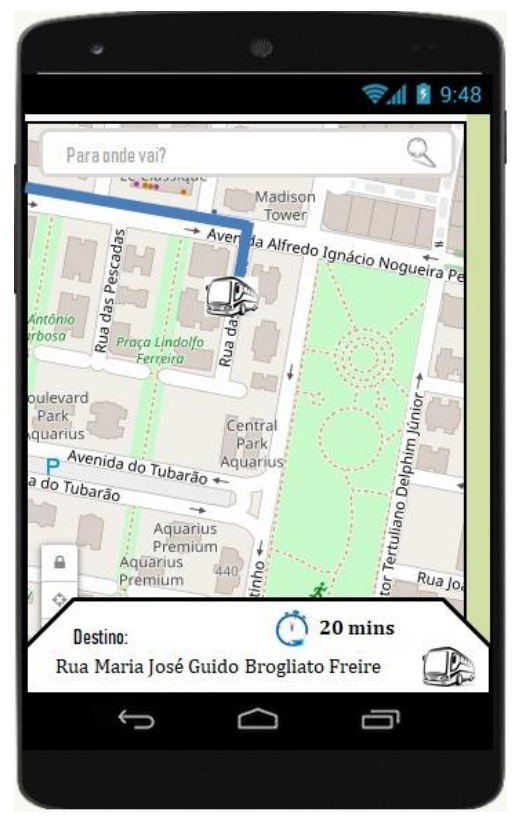

Tela 5. Aplicativo

\section{CONSIDERAÇÕES FINAIS}

Torna-se valido denotar que o transporte público coletivo poderá se tornar mais eficiência com a aplicação deste modelo de aplicativo, pois este torna as informações do transporte mais claras, e consegue integrar as viagens tendo em vista a necessidade de conexões da cidade, onde um usuário deve, diversas vezes, utilizar mais de um ônibus para chegar a seu destino mesmo dentro da cidade e isto, em geral, gera desconforto por não possuir a certeza de qual ônibus deverá utilizar quando finalizar a primeira parte do trajeto, isto inclui o horário de chegada do transporte e inclusive do ponto que deverá utilizar.

Neste caso, com a atendimento destas especificidades torna-se um interesse de valor da parte da população que não faz uso deste meio de transporte, parte fundamental para colaborar com os objetivos da cidade que busca meios de melhorar a locomoção e o fluxo das vias da cidade por meio do transporte coletivo.

Por fim, torna-se um investimento importante para o desenvolvimento da cidade, pois é notável o crescimento populacional, incluindo o número de veículos por pessoas na região, o que neste caso irá gerar com o passar do tempo um maior trafego e por conseguinte congestionamento nas vias urbanas o que as torna ineficientes, problema este que necessidade de ações imediatas para resolver ou ao menos criar alternativas.

\section{REFERÊNCIAS}

ANDRADE, C.; CUNHA, O. Pesquisa mobilidade da população urbana 2017. Disponível em: https://www.ntu.org.br/novo/upload/Publicacao/Pub636397002002520031.pdf. Acesso em: $12 / 05 / 2019$. 
BISSARO, D. Z. Mobilidade urbana: o direito de ir e vir no município de Teixeira de Freitas-BA. Disponível em https://www.uniara.com.br/arquivos/file/eventos/2017/seppu/anais/bissaro.pdf Acesso em: 23/04/2019.

BRITO, A. S.; PEREIRA, D. L. Mobilidade urbana e planejamento sustentável no município de Resende: estudo de caso exploratório. Disponível em: https://app.uff.br/riuff/bitstream/1/5343/1/Ariane\%20Santos\%20-\%20David\%20de\%20Lima.pdf.

Acesso em: 14/05/2019.

CARDIAS, E. H.; VALENTE A. F.; OLIVEIRA JUNIOR I.; OLIVEIRA E. Mobilidade urbana e cibercultura: as dificuldades no transporte coletivo como oportunidade de negócio na Amazônia. Disponível em http://portalintercom.org.br/anais/nacional2017/resumos/R12-0834-1.pdf Acesso em: 19/05/2019.

CLARK S. F. P.; SOUZA P. R. M. R. O transporte público de qualidade como um direito previsto no código de defesa do consumidor. http://www.joinpp.ufma.br/jornadas/joinpp2017/pdfs/eixo11/otransportepublicopublicodequalidade comoumdireitoprevistonocodigodedefesadoconsumidor.pdf Acesso em: 24/04/2019.

COSTA, C. A. Cidades inteligentes e mobilidade urbana. Disponível em https://fgvprojetos.fgv.br/sites/fgvprojetos.fgv.br/files/cadernos_fgvprojetos_smart_cities_bilinguefinal-web_0.pdf Acesso em: 27/02/2019.

GARCIA, G. F. O planejamento da cidade e a mobilidade urbana, uma combinação sustentável. Disponível em http://repositorio.unb.br/bitstream/10482/31814/5/2018_GeraldoFreireGarcia.pdf Acesso em: 23/04/2019.

GERHARDT, T. E.; SILVEIRA, D. T. Métodos de pesquisa. Disponível em http://www.ufrgs.br/cursopgdr/downloadsSerie/derad005.pdf Acesso em: 12/03/2019.

HINO, M. C.; CUNHA, M. A. V. C. Mobilidade urbana: uma abordagem de gênero e tecnologia no uso de aplicativos móveis. Disponível em https://singep.org.br/7singep/resultado/221.pdf Acesso em: 19/05/2019.

Instituto Pólis. Conheça o anteprojeto de lei da política nacional de mobilidade urbana Mobilidade urbana é desenvolvimento urbano. Disponível em http://www.polis.org.br/uploads/922/922.pdf Acesso em: 28/02/2019.

LIMA, C. C. B. Aplicativos móveis de interesse público: limites e possibilidades para a cidadania no Brasil.

Disponível

em http://repositorio.unb.br/bitstream/10482/23699/1/2017_C\%C3\%ADntiaCaldasBarcelardeLima.pdf Acesso em: 06/05/2019.

LOMBARDO, A; CARDOSO, O. R.; SOBREIRA, P. E. Mobilidade e sistema de transporte coletivo. Disponível em: http://www.opet.com.br/faculdade/revista-cc-adm/pdf/n7/MOBILIDADE-ESISTEMA-DE-TRANSPORTE-COLETIVO.pdf. Acesso em: 13/05/2019.

MARRARA, T. Transporte público e desenvolvimento urbano: aspectos jurídicos da política nacional de mobilidade. Disponível em: http://www.egov.ufsc.br:8080/portal/sites/default/files/84691127318-2-pb-3.pdf. Acesso em: 15/05/2019.

PARENTE, L. A.; MELO, P. G. S.; MACHADO, J. A.; NEVES, A. P. O planejamento como ferramenta de gestão para mobilidade urbana em um município do extremo norte do brasil. Disponível em https://singep.org.br/5singep/resultado/556.pdf Acesso em: 22/04/2019.

REIS, M. Mobilidade urbana: um desafio para gestores públicos. Disponível em: http://bibliotecadigital.fgv.br/dspace/bitstream/handle/10438/16446/cidades_inteligentes_e_mobilid ade_urbana_0.pdf?sequence=1\&isAllowed=y. Acesso em: 30/04/2019.

RUBIM, B; LEITÃO, S. O plano de mobilidade urbana e o futuro das cidades. Disponível em: http://www.scielo.br/scielo.php?script=sci_arttext\&pid=S0103-40142013000300005. Acesso em: 27/03/2019. 


\section{ISSN $2447-5378$}

SILVEIRA, M. R.; COCCO, R. G. Transporte público, mobilidade e planejamento urbano: contradições essenciais.

Disponível

em: http://www.scielo.br/scielo.php?script=sci_arttext\&pid=S0103-40142013000300004. Acesso em: 02/04/2019.

VIANNA G. S. B. Mobilidade urbana no Brasil: uma estimativa do produto perdido em trânsito. Disponível

https://fgvprojetos.fgv.br/sites/fgvprojetos.fgv.br/files/cadernos_fgvprojetos_smart_cities_bilinguefinal-web_0.pdf Acesso em: 27/02/2019. 